\title{
Distinct Phospho-Forms of Cortactin Differentially Regulate Actin Polymerization and Focal Adhesions
}

\author{
Anne E. Kruchten \\ Linfield College \\ Eugene W. Krueger \\ Mayo Clinic \\ Yu Wang \\ University of North Carolina at Chapel Hill \\ Mark A. McNiven \\ Mayo Clinic
}

Follow this and additional works at: https://digitalcommons.linfield.edu/biolfac_pubs

Part of the Cellular and Molecular Physiology Commons, and the Molecular Biology Commons

\author{
DigitalCommons@Linfield Citation \\ Kruchten, Anne E.; Krueger, Eugene W.; Wang, Yu; and McNiven, Mark A., "Distinct Phospho-Forms of \\ Cortactin Differentially Regulate Actin Polymerization and Focal Adhesions" (2008). Faculty Publications. \\ Published Version. Submission 6. \\ https://digitalcommons.linfield.edu/biolfac_pubs/6
}

This Published Version is protected by copyright and/or related rights. It is brought to you for free via open access, courtesy of DigitalCommons@Linfield, with permission from the rights-holder(s). Your use of this Published Version must comply with the Terms of Use for material posted in DigitalCommons@Linfield, or with other stated terms (such as a Creative Commons license) indicated in the record and/or on the work itself. For more information, or if you have questions about permitted uses, please contact digitalcommons@linfield.edu. 


\title{
Distinct phospho-forms of cortactin differentially regulate actin polymerization and focal adhesions
}

\author{
Anne E. Kruchten,* Eugene W. Krueger,* Yu Wang, and Mark A. McNiven \\ Department of Biochemistry and Molecular Biology and the Miles and Shirley Fiterman Center for Digestive Diseases, \\ Mayo Clinic, Rochester, Minnesota
}

Submitted 1 May 2008; accepted in final form 28 August 2008

\begin{abstract}
Kruchten AE, Krueger EW, Wang Y, McNiven MA. Distinct phospho-forms of cortactin differentially regulate actin polymerization and focal adhesions. Am J Physiol Cell Physiol 295: C1113-C1122, 2008. First published September 3, 2008; doi:10.1152/ajpcell.00238.2008.Cortactin is an actin-binding protein that is overexpressed in many cancers and is a substrate for both tyrosine and serine/threonine kinases. Tyrosine phosphorylation of cortactin has been observed to increase cell motility and invasion in vivo, although it has been reported to have both positive and negative effects on actin polymerization in vitro. In contrast, serine phosphorylation of cortactin has been shown to stimulate actin assembly in vitro. Currently, the effects of cortactin serine phosphorylation on cell migration are unclear, and furthermore, how the distinct phospho-forms of cortactin may differentially contribute to cell migration has not been directly compared. Therefore, we tested the effects of different tyrosine and serine phospho-mutants of cortactin on lamellipodial protrusion, actin assembly within cells, and focal adhesion dynamics. Interestingly, while expression of either tyrosine or serine phosphomimetic cortactin mutants resulted in increased lamellipodial protrusion and cell migration, these effects appeared to be via distinct processes. Cortactin mutants mimicking serine phosphorylation appeared to predominantly affect actin polymerization, whereas mutation of cortactin tyrosine residues resulted in alterations in focal adhesion turnover. Thus these findings provide novel insights into how distinct phospho-forms of cortactin may differentially contribute to actin and focal adhesion dynamics to control cell migration.
\end{abstract}

cell migration; phosphorylation

CORTACTIN IS AN ACTIN-BINDING protein that is a major substrate of $\mathrm{v}$-Src in transformed cells and a regulator of actin dynamics in response to various signaling pathways $(6,16)$. It is known to participate in a wide variety of actin-based cellular processes by supporting the regulated assembly of dynamic, branched actin networks that act to push and deform cellular membranes such as during lamellipodial protrusion or propulsion of vesicles via actin comet tails $(22,31,35)$. As a dynamic scaffolding protein, cortactin contains multiple interactive domains that bind to proteins involved in actin polymerization (see Fig. 1). These include an $\mathrm{NH}_{2}$-terminal acidic domain that binds to and activates the actin-related protein (Arp)2/3 complex (31) and a $\mathrm{COOH}$-terminal Src-homology 3 (SH3) domain that binds to, among other proteins, the actin-regulatory protein neural WiskottAldrich syndrome protein (N-WASP) $(18,19)$. In addition, cortactin contains a series of F-actin-binding domains and a central domain rich in proline, serine, and threonine residues.

How complexes of cortactin and actin-regulatory proteins are temporally assembled and localized to mediate actin polymeriza-

\footnotetext{
* A. E. Kruchten and E. W. Krueger contributed equally to this work.

Address for reprint requests and other correspondence: M. A. McNiven, Dept. of Biochemistry and Molecular Biology, Mayo Clinic, 200 First St. SW, Guggenheim 1637, Rochester, MN 55905 (e-mail: mcniven.mark@mayo.edu).
}

tion and support a complex process such as lamellipodial extension is not yet clear. However, the effects of cortactin on actin assembly are thought to be regulated through phosphorylation of cortactin by both tyrosine and serine/threonine kinases $(6,16,35)$. Src-mediated phosphorylation of tyrosine residues near the SH3 domain of cortactin has been implicated in altering actin organization, allowing for the disassembly of large cytoplasmic bundles and subsequent formation of a finer lamellipodial meshwork that may facilitate cell migration (11). Early studies indicated that cortactin is also phosphorylated on serine/threonine residues $(30$, 36); indeed, cortactin has been demonstrated to be a substrate for extracellular signal-regulated kinase (ERK) at two serine residues present in the central domain of cortactin immediately upstream of the Src-phosphorylated tyrosine residues $(3,18)$ (see Fig. 1). Furthermore, phosphorylation of cortactin by ERK at these serine residues has been suggested to alter its structural conformation, resulting in an increased association with N-WASP and a marked increase in actin polymerization. In contrast, Src-mediated tyrosine phosphorylation abolished the stimulatory effects of cortactin on N-WASP-Arp2/3-induced actin polymerization (18). However, a recent study by Tehrani et al. (28) revealed that when the adaptor protein Nck1 was present along with N-WASP and Arp2/3, tyrosine-phosphorylated cortactin had a positive effect on the generation of actin filament barbed ends in vitro.

A current gap in our understanding of cortactin function is an apparent disconnect between some of the observations made using in vitro assays, such as the negative effects of Srcmediated phosphorylation of cortactin on actin assembly, and those made in living cells. For example, endothelial cells overexpressing cortactin were observed to be exceptionally motile, while migration of cells into a wound was inhibited in cells expressing a cortactin mutant unable to be phosphorylated on key tyrosine residues (11). Similarly, overexpression of cortactin in breast cancer cells resulted in increased bone metastasis when these cells were injected into a nude mouse model, whereas injection of cells expressing a tyrosine phospho-inhibitory cortactin mutant resulted in fewer bone metastases as compared with the control group (14). Thus, the functions of cortactin appear to be regulated in a complex manner through phosphorylation at multiple sites. Indeed, a recent mass spectrometry analysis of cortactin purified from human embryonic kidney HEK-293 cells identified 17 new sites of cortactin phosphorylation for a total of 22 sites (4 tyrosine, 14 serine, and 4 threonine) when combined with the previously identified phosphorylation sites (17). However, the functional implications of this differential phosphorylation on

The costs of publication of this article were defrayed in part by the payment of page charges. The article must therefore be hereby marked "advertisement" in accordance with 18 U.S.C. Section 1734 solely to indicate this fact. 
actin dynamics with relation to migration of living cells need to be tested and compared.

Here, using the expression of phospho-mimetic and phospho-inhibitory cortactin tyrosine and serine mutants in cultured tumor cells and fibroblasts, we provide a detailed analysis of the mechanisms by which serine and tyrosine phosphorylation of cortactin may differentially contribute to cell migration. Interestingly, while phosphorylation at either site promotes cell migration, tyrosine phosphorylation appears to increase focal adhesion dynamics and lead to stress fiber disassembly. In contrast, serine phosphorylation of cortactin promotes the assembly of extensive branched actin networks. Thus these findings provide novel insights into how distinct types of phosphorylation may differentially regulate the effects of cortactin on specific processes to mediate cell migration and metastasis.

\section{MATERIALS AND METHODS}

Cells. Human pancreatic tumor cells (HPAF-II, BxPC-3, and PANC1), rat fibroblasts (FR), and normal rat liver cells (Clone 9) were acquired from American Type Culture Collection (Rockville, MD). Mouse embryonic fibroblasts (MEFs) were a gift from András Kapus (University of Toronto, Toronto, Canada). HPAF-II cells were maintained in EMEM (Mediatech, Herndon, VA) supplemented with $0.1 \mathrm{mM}$ nonessential amino acids and $1 \mathrm{mM}$ sodium pyruvate; BxPC-3 cells were maintained in RPMI 1640 (Mediatech) supplemented with glutamine, $10 \mathrm{mM}$ HEPES, $4.5 \mathrm{mM}$ glucose, and $1.5 \mathrm{mM}$ sodium bicarbonate; PANC-1, FR, and MEF cells were maintained in DMEM (Mediatech); and Clone 9 cells were maintained in Ham's F12K medium (Mediatech). Media for all cell lines contained $10 \% \mathrm{FBS}$, and $100 \mathrm{U} / \mathrm{ml}$ penicillin and $100 \mu \mathrm{g} / \mathrm{ml}$ streptomycin (Invitrogen, Carlsbad, CA). All cells were grown at $37^{\circ} \mathrm{C}$ in $5 \% \mathrm{CO}_{2}$. Cells were cultured on normal or gridded acid-washed glass coverslips.

Constructs, transfection, and other reagents. Cells were transfected with the indicated constructs using either the Lipofectamine Plus Transfection Reagent (Invitrogen) or Genejammer Transfection Reagent (Stratagene, La Jolla, CA) according to the manufacturer's instructions. The cDNA sequence corresponding to rat cortactin isoform $\mathrm{B}$ (GenBank accession no. NM_021868) was used for generating wildtype and phospho-mutant cortactin constructs. Generation of wildtype red fluorescent protein (RFP)-tagged cortactin has been previously described (4). RFP-cortactin tyrosine and serine mutants were generated on the basis of known tyrosine (11) and serine $(3,18)$ phosphorylation sites using the Stratagene QuikChange Site-Directed Mutagenesis kit (Stratagene). The Myc-tagged type 1 phosphatidylinositol-4-phosphate 5-kinase- $\alpha$ (PIP5K1 $\alpha$ ) construct was a gift from Laura M. Machesky (University of Birmingham, Birmingham, UK), and the green fluorescent protein (GFP)-paxillin construct was a gift from Christopher E. Turner (State University of New York, Syracuse, NY). Monoclonal anti-paxillin antibody was from BD Biosciences (San Jose, CA), monoclonal anti-vinculin antibody was from Sigma (St. Louis, MO), and FITC-conjugated phalloidin and Alexaconjugated secondary antibodies were from Invitrogen. EGF was from Invitrogen, and nocodazole was from Sigma.

Microscopy. Cells were processed for fluorescence microscopy as previously described (10), except that cells were fixed with $4 \%$ formaldehyde and permeabilized with $0.1 \%$ Triton $\mathrm{X}-100$ for 2 to $5 \mathrm{~min}$, depending on the cell type. ProLong (Invitrogen) antifade reagent was used as mounting medium. Live imaging experiments were performed using previously described conditions (21). Microscopy was performed on a Zeiss Axiovert 200 epifluorescent microscope (Zeiss, Thornwood, $\mathrm{NJ})$. Digital images were acquired and analyzed using a cooled-CCD Orca II camera (Hamamatsu Photonics, Hamamatsu City, Japan) and the software program IPLab (Scanalytics, Fairfax, VA). All images were processed using Adobe Photoshop software (Adobe Systems, Mountain View, CA).

To analyze comets using electron microscopy, cells containing PIP5K1 $\alpha$-induced comets positive for RFP-tagged cortactin were first identified using fluorescence microscopy. These cells were then processed for electron microscopy analysis using standard procedures and observed with a JEOL 1200 transmission electron microscope (JEOL USA, Peabody, MA).

Lamellipodial protrusion. Transfected cells were plated on glass coverslips and serum starved (0.2\% FBS) for $16 \mathrm{~h}$. Subsequently, cells were either mock stimulated with carrier or stimulated with EGF (30 $\mathrm{ng} / \mathrm{ml}$ ) for $20 \mathrm{~min}$, processed for fluorescence microscopy analysis, and stained with FITC-conjugated phalloidin. The percentage of transfected cells extending a lamellipodium was determined for each condition.

Wound healing. Transfected PANC-1 cells were grown to confluency on gridded glass coverslips, serum starved ( $0.2 \% \mathrm{FBS})$ for $16 \mathrm{~h}$, and then wounded with a pipette tip. After thorough washing, low serum medium was replaced with full serum medium, and the migration of cells into the wound was followed over a 14-h time period. At time 0 , multiple images of each slip were taken, focusing on the coverslip grid. At regular time intervals afterward, images were taken in the exact same locations using the grid as a reference to track the migration of cells in the wound. Image integration was performed using IPLab software, and individual fluorescent cells were tracked. All fluorescent cells within a $100-\mu \mathrm{m}$ distance from the wound front at time 0 were tracked. Approximately 20 cells were analyzed for each condition. The total distance each cell traveled was measured, and since under some conditions the cells traveled in aberrant pathways, the net distance into the wound perpendicular to the wound front was also measured.

Comet formation. FR cells were plated on glass coverslips and were cotransfected with RFP-cortactin and Myc-PIP5K1 $\alpha$ constructs. Twenty-four hours after transfection, cells were processed for fluorescence microscopy analysis, staining with FITC-conjugated phalloidin, or correlative electron microscopy analysis. Additionally, transfected cells were plated in imaging dishes, and comets were imaged in living cells via the RFP-cortactin in their tails.

Focal adhesion dynamics. Focal adhesion size and morphology were assessed in fixed FR cells that had been allowed to spread for $6 \mathrm{~h}$ on coverslips coated with $1 \mu \mathrm{g} / \mathrm{ml}$ fibronectin (Calbiochem, San Diego, $\mathrm{CA}$ ) by staining with a monoclonal anti-paxillin antibody or on the basis of GFP-paxillin signal.

To monitor focal adhesion turnover in living cells, MEFs and PANC-1 cells were cotransfected with GFP-paxillin and RFP-cortactin constructs. Twenty-four hours after transfection, cells were trypsinized and resuspended in serum-containing medium. Subsequently, cells were replated on coverslips coated with $1 \mu \mathrm{g} / \mathrm{ml}$ fibronectin and allowed to spread for $30 \mathrm{~min}$ before being imaged using either time-lapse confocal microscopy or total internal reflection fluorescence (TIRF) microscopy.

Focal adhesion reassembly following microtubule-mediated focal adhesion disassembly was assayed in MEFs and PANC-1 cells as follows. First, cells were treated with $10 \mu \mathrm{g} / \mathrm{ml}$ nocodazole for $3 \mathrm{~h}$ at $37^{\circ} \mathrm{C}$ to induce microtubule disassembly. The drug was then washed out, and microtubules were allowed to repolymerize over various time intervals. Subsequently, cells were fixed and processed for immunofluorescence, staining with a monoclonal antibody against vinculin. Cells were imaged using fluorescence microscopy, and the presence or absence of focal adhesions was quantitated.

\section{RESULTS}

Cortactin phosphorylation promotes lamellipodial protrusion and cell migration. We have previously observed that cortactin is present at both plasma membrane clathrin-coated pits and the Golgi in cultured rat hepatocytes (Clone 9) $(4,5)$ 
(Fig. 1A), an epithelial cell type normally exhibiting a discoidal cell shape. Interestingly, expression of a RFP-tagged version of cortactin mutated to mimic Src-mediated phosphorylation (rat cortactin isoform b Y384/429/445E; CortYE) in these cells resulted in graphic changes in cell morphology. Even in the absence of growth factor stimulation, Clone 9 cells expressing CortYE appeared elongated and highly polarized, and extended elaborate lamellipodia (Fig. 1B). These initial observations made in nonmotile, normal epithelial cells provided a strong incentive to test the effects of the different phospho-forms of cortactin on growth factor-mediated induction of lamellipodial extension and migration in inherently motile cell types, particularly cancer cells. Toward this end, we compared the effects of tyrosine versus serine cortactin phospho-mutants on cell migration, the assembly of actin structures in the confines of living cells, and focal adhesion dynamics using cultured fibroblasts (MEFs and RF cells) and three human pancreatic ductal carcinoma cell lines, BxPC-3 (27), PANC-1 (15), and HPAF-II $(12,24)$. (For a graded comparison of these lines, see Ref. 26.)

In addition to generating the RFP-tagged cortactin mutant mimicking Src-mediated phosphorylation (CortYE) indicated above, cortactin phospho-mutants designed to inhibit (Y384/ 429/445F; CortYF) Src-mediated phosphorylation, or inhibit (S368/381A; CortSA) or mimic (S368/381D; CortSD) ERKmediated phosphorylation were constructed on the basis of previously identified sites of tyrosine (11) and serine $(3,18)$ phosphorylation. These mutants, RFP-tagged wild-type cortactin (CortWT) or vector alone, were then expressed in BxPC-3 (Fig. $1, C-G$ ) or HPAF-II cells (supplemental material Fig. S1; the online version of this article contains supplemental data), and the effects on the percentage of cells that formed lamellipodia in the absence and presence of EGF were quantitated. As expected, EGF treatment resulted in an approximately 3.5 -fold stimulation of lamellipodial protrusion in vector- and CortWTexpressing cells (Fig. $1 H$ and supplemental Fig. S1F). Interestingly, cells expressing the cortactin phospho-mimetic mutants CortSD and CortYE exhibited an approximately 2.5- to 3 -fold increase in the percentage of cells that extended lamellipodia in the absence of EGF, as compared with control and CortWT-expressing cells (Fig. $1 H$ and supplementary Fig. $\mathrm{S} 1 F$; gray bars). Furthermore, this EGF-dependent extension of lamellipodia was reduced in cells expressing the cortactin

\section{Cortactin}
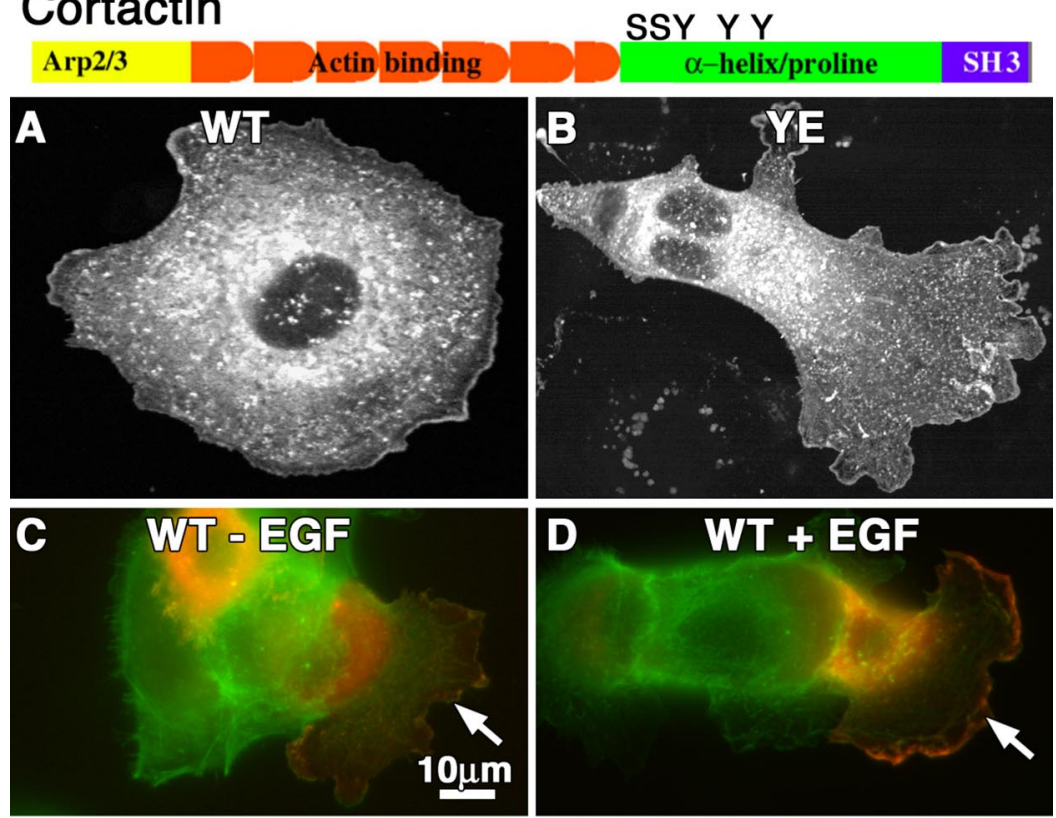

Fig. 1. Expression of phospho-mimetic forms of cortactin induces a dramatic motile phenotype in nonmigratory epithelial cells and in pancreatic tumor cells. A cartoon of the cortactin protein is shown with specific protein domains and sites of serine (S) and tyrosine (Y) phosphorylation relevant to the studies indicated. SH3, COOH-terminal Src-homology 3 domain; Arp, actin-related protein. $A$ and $B$ : fluorescence images of cultured hepatocytes (Clone 9 cells) expressing red fluorescent protein (RFP)-tagged wild-type cortactin (CortWT; $A$ ) or an RFP-tagged cortactin mutant mimicking tyrosine phosphorylation (Y384/429/445E, CortYE; $B$ ). Clone 9 cells expressing CortWT are discoidal in shape and exhibit a modest amount of peripheral membrane ruffling $(A)$. In contrast, expression of CortYE in these cells induces marked changes in cell morphology, as they become elongated and extend elaborate lamellipodia $(B) . C-G$ : fluorescence images of BxPC-3 pancreatic tumor cells that were transfected with either vector alone, as a control, or RFP-tagged cortactin constructs (red), serum starved overnight, and when indicated, treated with EGF (30 ng/ml) for 20 min. Subsequently, cells were processed for analysis by fluorescence microscopy and were stained with FITC-conjugated phalloidin (green) to label actin filaments. Cells expressing CortWT extended lamellipodial protrusions under serumstarved conditions $(C$, arrow) at a frequency similar to that of cells expressing vector alone (data not shown), and this was increased upon EGF treatment ( $D$, arrow). Lamellipodial protrusion (arrows) was increased by EGF treatment in untransfected cells, but a cell expressing a cortactin mutant inhibiting tyrosine phosphorylation (CortYF; $E$, arrowhead) shows virtually no lamellipod. Conversely, cells expressing cortactin mutants mimicking either serine phosphorylation (CortSD; $F$ ) or tyrosine phosphorylation (CortYE; $G$ ) frequently extended lamellipodia (arrows) even in the absence of EGF. $H$ : graph representing the percentage of cells expressing vector alone $(\mathrm{V})$, CortWT, or cortactin phospho-mutants that extended lamellipodia in the absence (gray bar) and presence (black bar) of EGF. SA, cortactin mutant inhibiting serine phosphorylation. Values represent the average \pm SE for $n=3$ with $>100$ cells counted for each condition. Bar, $10 \mu \mathrm{m}(A-G)$.

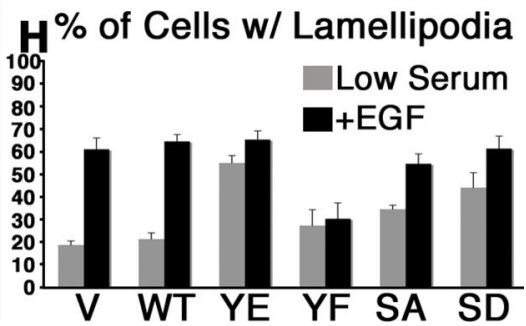

AJP-Cell Physiol • vOL 295 • NOVEMBER $2008 \cdot$ www.ajpcell.org 
phospho-inhibitory mutant CortYF. The baseline lamellipodial formation seen in cells expressing the phospho-inhibitory CortSA mutant may be attributed to the prolonged activation of ERK caused by overexpression of cortactin as demonstrated by Timpson et al. (29).

We next tested whether the increase in lamellipodial protrusion exhibited by the cortactin tyrosine and serine phosphomimetic mutants (CortSD and CortYE) may translate into increased cell motility. We observed PANC-1 cells to be more motile than HPAF-II or BxPC-3 cells (A. E. Kruchten, E. W. Krueger, and M. A. McNiven, unpublished observations); thus, wound-healing assays were performed using PANC-1 cells. Expression of CortWT enhanced the migratory phenotype of cells (Fig. 2, $A$ and $D$ ), in agreement with previous reports that overexpression of cortactin in fibroblasts and endothelial cells enhances cell migration $(11,23)$. Similarly, cells expressing CortSD or CortYE also exhibited an enhancement in cell migration, although not above that of CortWT-expressing cells (Fig. 2, $C$ and $D$, and data not shown). In contrast, cells expressing CortSA or CortYF migrated only approximately half as far as cells expressing CortWT or their respective phospho-mimetic mutant (Fig. 2, $B$ and $D$, and data not shown). Thus, both tyrosine and serine phosphorylation of cortactin appear to be important not only for lamellipodial protrusion, but also for the stimulatory effects of cortactin on cell migration.

Serine phosphorylation of cortactin promotes actin assembly in living cells. Similar to the branched actin network formed at the leading edge of migrating cells, actin comets used to propel some intracellular vesicles and pathogens also exhibit a branched actin network $(2,9)$. Therefore, because it is challenging to obtain quantitative measurements of actin assembly at lamellipodia in living cells, we analyzed vesicleassociated actin comet tails that are generated in cultured cells (here, FR cells) upon overexpression of the lipid kinase type 1 phosphatidylinositol-4-phosphate 5-kinase- $\alpha$ (PIP5K1 $\alpha ; 20$, $25)$. These comets are easily visualized and act to push vesicles forward using an actin network also containing cortactin (20). Thus we could readily quantify the number and dimensions of actin comet tails formed behind vesicles in fixed or living cells coexpressing Myc-tagged PIP5K1 $\alpha$ and either wild-type or phospho-mutants of RFP-tagged cortactin based on the fluo- rescent cortactin incorporated into the tail and/or FITC-conjugated phalloidin, used to label actin. Most notably, in comparison to the number of comets formed in cells expressing CortWT (Fig. 3A) or CortSA (data not shown), CortSDexpressing cells (Fig. 3C) showed a substantial increase in comet number $(\sim 2$-fold; Fig. $3 J)$, suggesting an increase in actin polymerization. In contrast, expression of cortactin tyrosine mutants, either phospho-inhibitory (CortYF; Fig. 3B) or phospho-mimetic (CortYE; data not shown), only slightly increased the number of comets per cell in comparison to CortWTexpressing cells (Fig. 3J).

Just as comet number can be used as an indicator of sites of actin polymerization, the lengths and widths of individual actin comet tails may also be representative of increased actin branching and polymerization. The lengths of comet tails was similar in cells expressing CortWT, CortYE, and CortSD, possibly indicating that actin polymerization and branching following nucleation is affected in a similar way by cortactin tyrosine and serine phosphorylation. Indeed, comet tail length was decreased in cells expressing either CortYF or CortSA, as compared with CortWT- or cortactin phospho-mimetic-expressing cells (Fig. 3K). In contrast, comet tail width was decreased in cells expressing CortYF or CortYE (Fig. $3 L$ ), but it was increased in cells expressing CortSD (Fig. 3, $D^{\prime}$ and $L$ ). Interestingly, many of the comets generated in the CortSDexpressing cells were so exceptionally thick as to appear bifurcated and composed of two parallel, bright actin bundles (Fig. 3 $D^{\prime}$ ), a phenotype not observed in cells expressing CortWT (Fig. 3D).

To confirm the differences in actin comet tail dimensions observed in cells expressing the different cortactin phosphomutants at a higher resolution, comets were viewed using electron microscopy (Fig. 3, E-I). In support of the fluorescence imaging, the shorter, thinner comets formed in the CortSA-expressing cells exhibited an actin network that appeared to surround only a small portion of the vesicle ( $\sim 30-40 \%$; Fig. 3, $F$ and $G$ ), whereas in cells expressing CortSD, a larger portion of the vesicle was surrounded by actin filaments ( $\sim 50-70 \%$; Fig. 3 , $H$ and $I$ ). In addition, the comet base was wider in cells expressing CortSD, as compared with cells expressing CortSA.
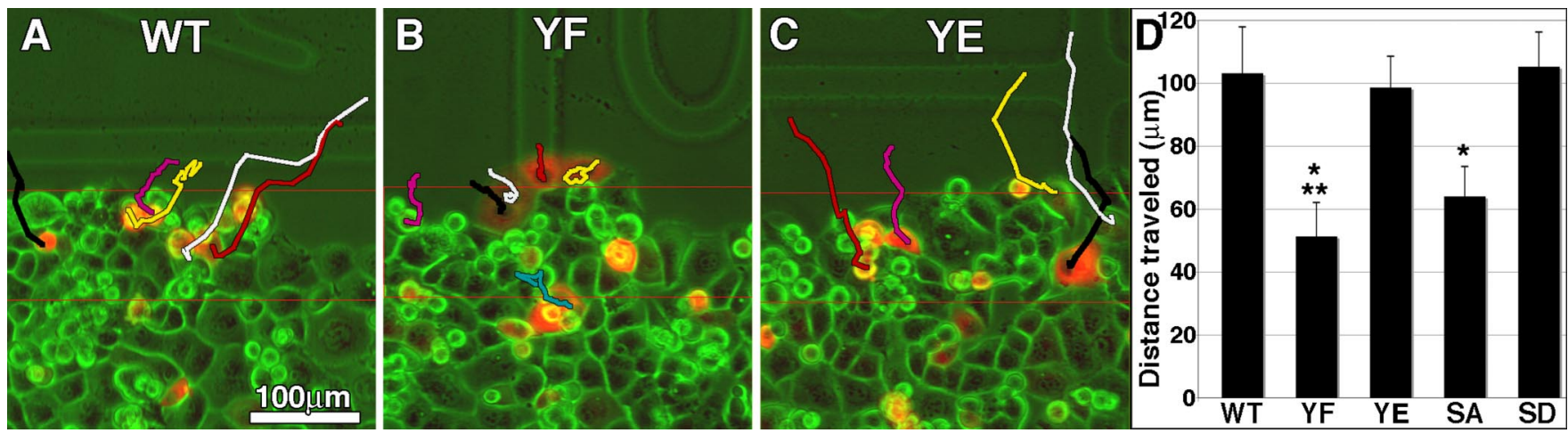

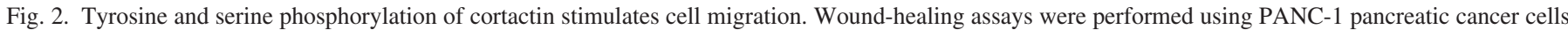

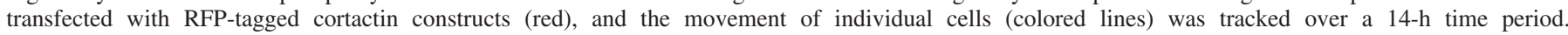

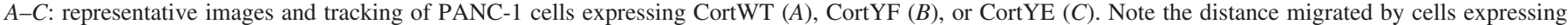

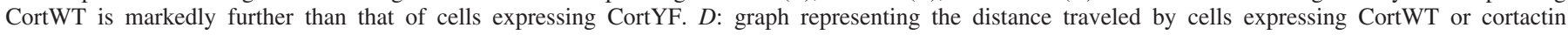

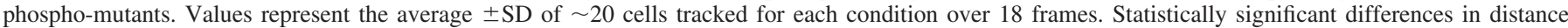

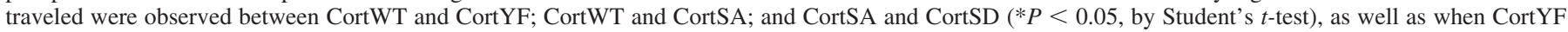
is compared with either CortYE or CortSD $(* * P<0.01)$. Bar, $100 \mu \mathrm{m}(A-C)$. 

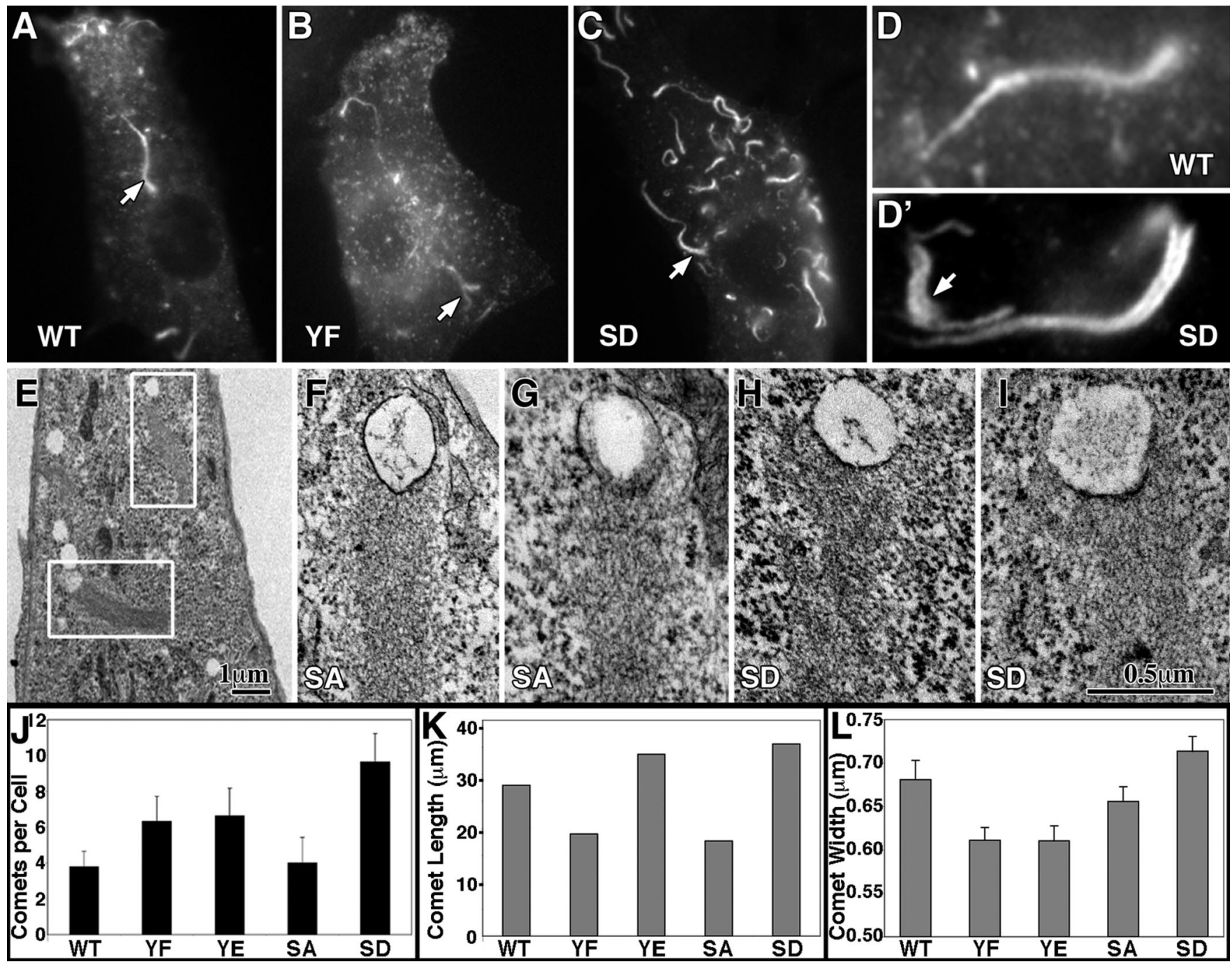

Fig. 3. Serine phosphorylation of cortactin increases the number, length, and caliber of PIP5K1 $\alpha$-induced comets. Rat fibroblasts (FR cells) coexpressing Myc-PIP5K1 $\alpha$, to induce comet formation, and RFP-tagged wild-type or phospho-mutant cortactin were analyzed using fluorescence microscopy and electron microscopy, quantitating the number of comets per cell and comet tail length and width. Comet tails were observed and measured by using the cortactin RFP signal as a marker. $A-C$ : cells expressing CortWT $(A)$ or CortYF $(B)$ had fewer and shorter comets than cells expressing CortSD $(C)$. $D$ and $D^{\prime}$ : some of the comets in the CortSD-expressing cells appeared to be substantially wider at the base $\left(D^{\prime}\right)$, compared with comets in CortWT-expressing cells $(D)$. $E-I$ : electron micrographs of PIP5K1 $\alpha$-induced comets in cells expressing RFP-tagged CortWT ( $E$, low magnification), CortSA ( $F$ and $G$, high magnification), or CortSD $(H$ and $I$, high magnification). The actin tails in CortSA-expressing cells $(F$ and $G$ ) were narrower and did not extend as far around the lipid droplet as in CortSD-expressing cells $(H$ and $I) . J-L$ : graphs representing the number of fluorescent comets per living cell $(J)$, comet tail length $(K)$, and comet tail width $(L)$ in cells expressing CortWT or the various cortactin phospho-mutants. While the length of comet tails was increased both in CortYE- and CortSD-expressing cells, comet tail thickness appeared to be increased more so in cells expressing CortSD. Values represent the average \pm SE of $\sim 50$ comets from at least 10 cells analyzed for comet number and length for each condition $(J$ and $K)$, and of 55-115 comets from 6 to 8 cells analyzed for comet width for each condition $(L)$. Statistically significant differences in comet number $(J)$ were observed between CortWT and CortSD; and CortSA and CortSD $(P<0.05)$. Differences in comet lengths $(K)$ were not statistically significant. Statistically significant differences in comet widths $(L)$ were observed between CortSD and CortYF; and CortSD and CortYE $(P<0.05)$. Bars, $1 \mu \mathrm{m}(E)$ and $0.5 \mu \mathrm{m}(F-I)$.

Tyrosine phosphorylation of cortactin alters focal adhesion morphology and dynamics. Observation of actin comet tails indicated that serine phosphorylation of cortactin preferentially promotes actin assembly in cells; however, both tyrosine and serine phospho-mimetic cortactin mutants promote lamellipodial protrusion and cell migration (Figs. 1 and 2 and supplemental Fig. S1). This paradox suggests that Src-mediated tyrosine phosphorylation of cortactin may be contributing to these dynamic processes in a way distinct from actin assembly. Some insights into this possibility come from a recent study showing that knockdown of cortactin protein levels reduces the rate of focal adhesion assembly (1) and our observation that some cells expressing cortactin appear to exhibit prominent focal contact-like structures (Fig. 6). Thus, because Src-mediated phosphorylation is known to play a role in regulating focal adhesion dynamics $(8,34)$, we tested whether tyrosine phosphorylation of cortactin may contribute to lamellipodial protrusion not by actin assembly at the leading edge, but by regulating focal adhesion turnover.

First, focal adhesion size was analyzed in two different cell types: FR cells, chosen because of the large adhesions made by 
these cells (Fig. 4A, $A^{\prime}$ ), and PANC-1 cells, which exhibit smaller, more ephemeral adhesions (Fig. $4 F, F^{\prime}$ ). To mark focal adhesions, cells were transfected to express RFP-tagged CortWT or the cortactin phospho-mutants in combination with GFP-tagged paxillin, a widely used marker for monitoring focal adhesion dynamics $(32,34)$, or alternatively, cells were stained with an anti-paxillin antibody. As shown in Fig. 4, FR cells expressing CortYE exhibited small, sparsely populated focal adhesions (Fig. 4B, $B^{\prime}$ ), compared with cells expressing CortWT (Fig. 4A, $A^{\prime}$ ), whereas CortYF-expressing cells exhibited much larger, more densely packed focal adhesions that appeared to fill the cell border (Fig. $4 C, C^{\prime}$ ). Quantitation of focal adhesion size in FR cells expressing wild-type cortactin or the various cortactin phospho-mutants revealed a slight reduction in size in cells expressing CortYE, CortSA, or CortSD, as compared with CortWT-expressing cells, whereas expression of CortYF resulted in an increase in focal adhesion size (Fig. 4D). Indeed, a 2.5-fold difference in focal adhesion size was determined in cells expressing CortYF versus CortYE. While more difficult to quantitate, similar effects on focal adhesion size were observed in PANC-1 tumor cells expressing CortYE (Fig. 4G, $G^{\prime}$ ) and CortYF (Fig. 4H, $H^{\prime}$ ) compared with cells expressing CortWT (Fig. $4 F, F^{\prime}$ ).

Changes in focal adhesion size could be a reflection of changes in the dynamics of these structures. Therefore, focal adhesion turnover was monitored in the context of CortWT, CortYE, or CortYF expression by following the changes in fluorescence intensity of GFP-paxillin over time in a localized region on initiation of focal adhesion formation. For these studies, MEFs expressing GFP-paxillin and RFP-tagged wildtype or phospho-mutant cortactin were plated on coverslips coated with $1 \mu \mathrm{g} / \mathrm{ml}$ fibronectin and allowed to spread for 30 min before imaging of focal adhesions at the very base of cells using TIRF microscopy. As shown by the line graph of representative curves from individual MEFs, cells expressing CortWT contained focal adhesions that assembled in $\sim 10-15$
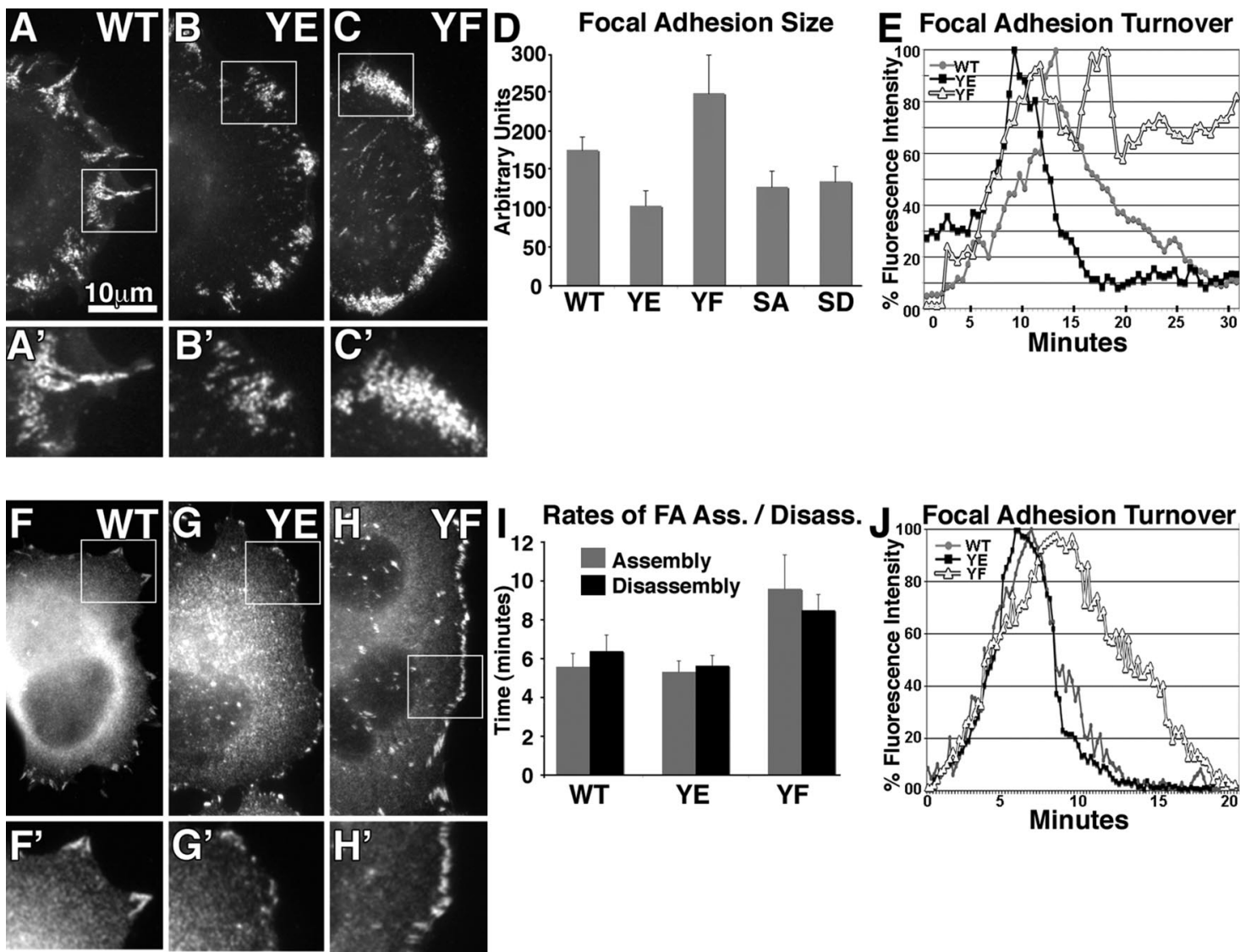

Fig. 4. Tyrosine phosphorylation of cortactin modulates focal adhesion size and stability. Focal adhesion size and dynamics were monitored in either FR cells, mouse embryonic fibroblasts (MEFs), or PANC-1 cells expressing RFP-tagged wild-type or phospho-mutant cortactin. Green fluorescent protein (GFP)-paxillin or staining with an anti-paxillin antibody was used as a marker for focal adhesions. $A, A^{\prime}$ : a representative image of focal adhesions in an FR cell expressing CortWT. $B-C^{\prime}$ : focal adhesions in FR cells expressing CortYE $\left(B, B^{\prime}\right)$ were much smaller than those in CortWT-expressing cells and composed of more discrete GFP-paxillin puncta, whereas the focal adhesions in cells expressing CortYF $\left(C, C^{\prime}\right)$ were markedly larger and more densely packed. $D$ : graph representing analysis of focal adhesion size in FR cells. A 2.5-fold difference exists between cells expressing CortYF versus those expressing CortYE. Values represent the average $\pm \mathrm{SE}$ of $\sim 10 \mathrm{FR}$ cells analyzed for each condition. $E$ : line graph of representative curves from individual MEFs expressing CortWT, CortYE, or CortYF, indicating changes in GFP-paxillin fluorescence intensity, and thereby focal adhesion assembly and disassembly, over time. $F-H^{\prime}$ : similar to FR cells, staining of PANC-1 cells expressing the cortactin tyrosine phospho-mutants (CortYE, $G, G^{\prime}$; and CortYF, $H, H^{\prime}$ ) with an antibody against paxillin also revealed differences in focal adhesion size, as compared with cells expressing CortWT $\left(F, F^{\prime}\right) . I$ : graph showing the amount of time required for focal adhesion (FA) assembly or disassembly in PANC-1 cells expressing CortWT, CortYE, or CortYF. Values represent the average \pm SE of approximately 6-8 PANC-1 cells analyzed for each condition. $J$ : line graph, similar to that shown in $E$; however, data from multiple PANC-1 cells were compiled to generate a single representative curve for each condition (expression of CortWT, CortYE, or CortYF). Bar, $10 \mu \mathrm{m}(A-C$ and $F-H)$; insets are higher magnifications of the respective boxed regions of paxillin at focal adhesions. 
min and subsequently almost completely disassembled over the following $\sim 5 \mathrm{~min}$ (Fig. $4 E$ ). Interestingly, CortYE-expressing cells assembled and disassembled their small focal adhesions much faster, as they both appeared and disassembled over an $\sim 15$-min time period. In strong contrast, the large focal adhesions present in CortYF-expressing cells assembled at a rate similar to that of CortWT-expressing cells, but persisted at $\sim 70 \%$ of the peak GFP-paxillin fluorescence intensity beyond 30 min (Fig. 4E). PANC-1 cells demonstrated focal adhesion dynamics similar to those of MEFs, with cells expressing CortYF taking $\sim 1.5-2$ times as long as CortWT-expressing cells to assemble or disassemble focal adhesions (Fig. 4I). Compilation of this focal adhesion assembly-disassembly data from multiple PANC-1 cells into a single representative curve for each condition (CortWT, CortYE or CortYF expression) provides another way of depicting that focal adhesion dynamics are slowed in PANC-1 cells expressing CortYF (Fig. 4J).

As a second method to assess a role for cortactin phosphorylation in regulating focal adhesion size and turnover, we used a microtubule-mediated focal adhesion disassembly assay similar to that established by Ezratty et al. (7). Here, MEFs and PANC-1 cells expressing wild-type or phospho-mutant cortactin were treated with nocodazole to disassemble microtubules and induce formation of focal adhesions. Subsequently, following washout of the drug, cells were allowed to recover for $2 \mathrm{~h}$ and were then stained for the focal adhesion marker vinculin. During the first $60 \mathrm{~min}$ of the recovery period, $>90 \%$ of the focal adhesions are disassembled, possibly by an endocytic mechanism (7), while focal adhesion reassembly occurs during the second $60 \mathrm{~min}$. It is predicted that ephemeral, dynamic focal adhesions will not form as quickly during the allotted recovery period, while stable adhesions will reassemble more readily. Consistent with this premise, both MEFs and PANC-1 cells expressing CortYE showed a marked decrease in focal adhesion recovery (Fig. 5, $A, A^{\prime}$ and $C, C^{\prime}$, respectively, asterisks) compared with the CortWT- or CortYF-expressing cells (Fig. 5, $B, B^{\prime}$ and $D, D^{\prime}$, asterisks; and data not shown). In contrast, focal adhesions in CortYF-expressing cells (Fig. 5,

Fig. 5. Expression of cortactin tyrosine phospho-mutants leads to distinct effects on the ability of cells to reassemble focal adhesions. $A-D^{\prime}$ : MEFs $\left(A-B^{\prime}\right)$ and PANC-1 cells $\left(C-D^{\prime}\right)$ expressing wild-type or tyrosine phosphomutant cortactin (asterisks) were treated with nocodazole for $3 \mathrm{~h}$ to disassemble microtubules and enhance formation of focal adhesions. Subsequently, following drug washout, cells were allowed to recover for $2 \mathrm{~h}$ and were then fixed and stained for vinculin (right). Additionally, cells were fixed and stained in the absence of any recovery period (left). In untransfected cells, microtubule regrowth induces focal adhesion disassembly in the first $60 \mathrm{~min}$, followed by focal adhesion reassembly over the next $60 \mathrm{~min}$. In MEFs and PANC-1 cells expressing CortYE (asterisks), focal adhesions were prominent before microtubule regrowth ( $A$ and $C$, arrows) and disassembled in the initial 60-min recovery period (data not shown). Following $120 \mathrm{~min}$ of recovery, although focal adhesions had reassembled in surrounding untransfected cells $\left(A^{\prime}\right.$ and $C^{\prime}$, arrows), focal adhesions did not readily reassemble in CortYE-expressing cells ( $A^{\prime}$ and $C^{\prime}$, asterisks). MEFs and PANC-1 cells expressing CortYF (asterisks) also displayed large focal adhesions before drug washout ( $B$ and $D$, arrows), which disassembled upon microtubule regrowth (data not shown). In contrast to cells expressing CortYE, however, CortYF-expressing cells appeared to fully reassemble large focal adhesions over the 120-min recovery period ( $B^{\prime}$ and $D^{\prime}$, arrows). E: graph representing the percentage of MEFs (gray bars) and PANC-1 cells (black bars) expressing either wild-type or tyrosine phosphomutant cortactin that contained focal adhesions $2 \mathrm{~h}$ after washout of nocodazole. Values represent the average $\pm \mathrm{SD}$ for $n=3$ with $>60$ cells analyzed for each condition. Bars, $8 \mu \mathrm{m}\left(A-D^{\prime}\right)$.
$B, B^{\prime}$ and $D, D^{\prime}$, asterisks) recovered more readily and were noticeably larger than those in adjacent control cells as well as those in the CortYE-expressing cells. Semiquantitative counting of the number of focal adhesions in these cell populations (Fig. 5E) suggested modest differences in focal adhesion reassembly between cells expressing wild-type cortactin and either of the cortactin tyrosine phospho-mutants, whereas a more noticeable difference existed between cells expressing CortYE versus CortYF.

Cortactin has been implicated in focal adhesion formation (1), and our observations suggest that the different phosphoforms of cortactin exert marked effects on focal adhesion size and turnover in both normal and pancreatic tumor cells (Figs. 4 and 5). However, a direct localization of cortactin to these
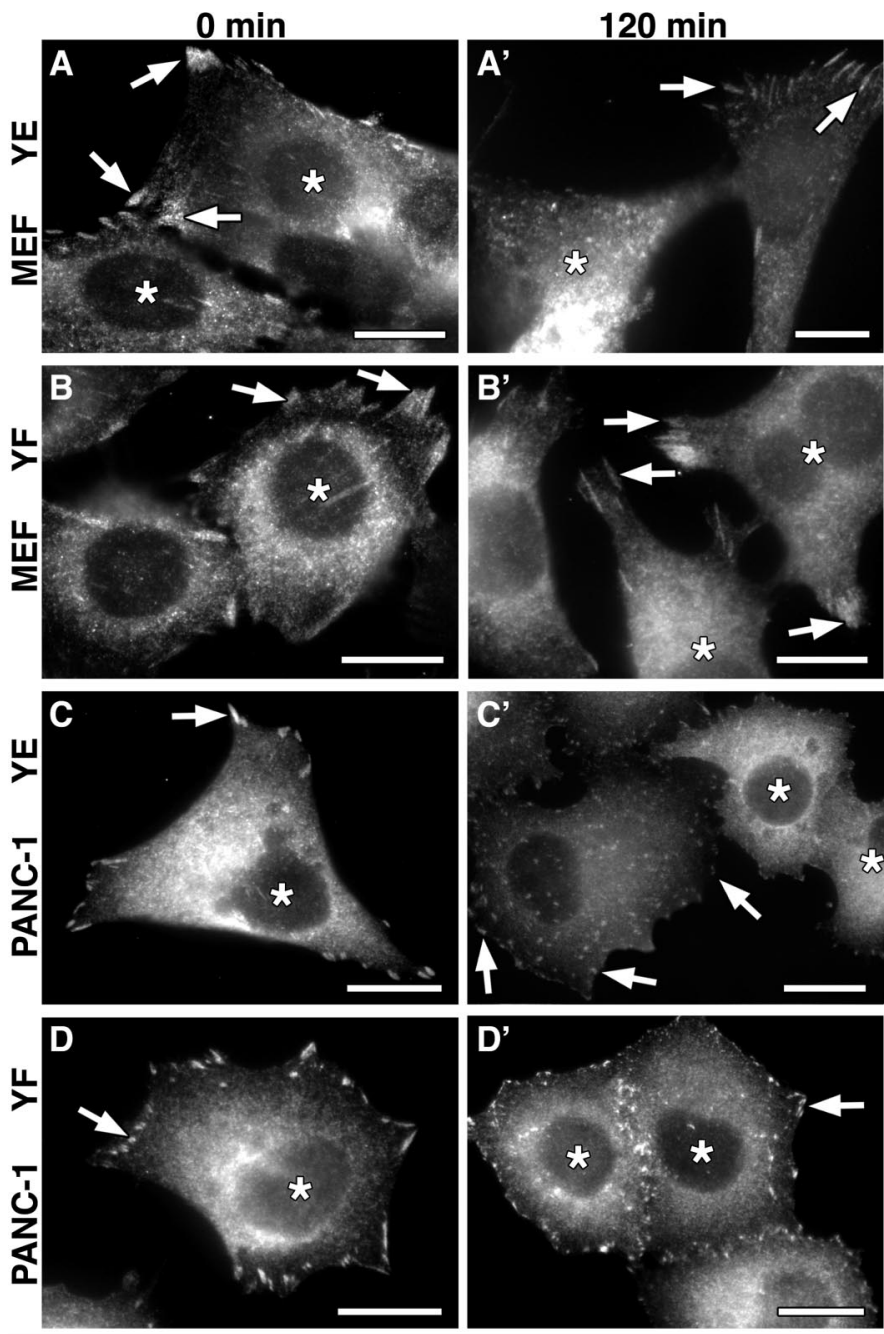

$\mathrm{E}_{60} \%$ of Cells w/ Focal Adhesions (2hr post washout)

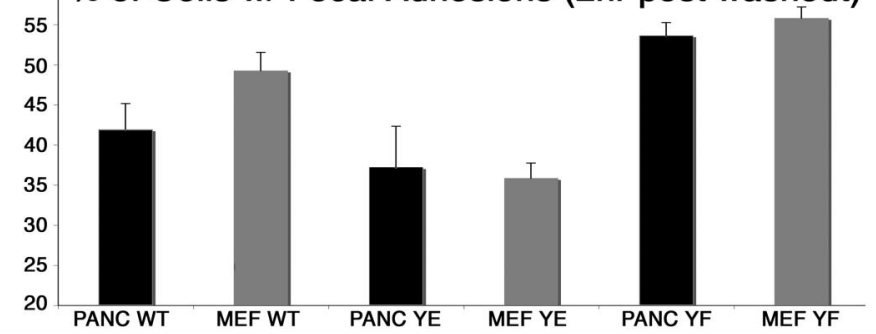


structures in cells is lacking, suggesting that cortactin could be affecting focal adhesions indirectly. As shown in Fig. 6, we detected a marked localization of CortWT to the tips of actin stress fibers in FR cells, which may represent sites of insertion into focal adhesions (Fig. 6A, $A^{\prime}$, arrows). Indeed, subsequent staining of cells with an antibody against paxillin confirmed that the CortWT-positive structures were focal adhesions (Fig. $6 B, B^{\prime}$, arrows). As discussed below, the action of tyrosinephosphorylated cortactin on focal adhesion stability and turnover could have profound effects on actin stress fiber dynamics and thereby markedly retard or accelerate cell migration.

\section{DISCUSSION}

The effects of tyrosine and serine/threonine phosphorylation of cortactin on actin polymerization have been observed using
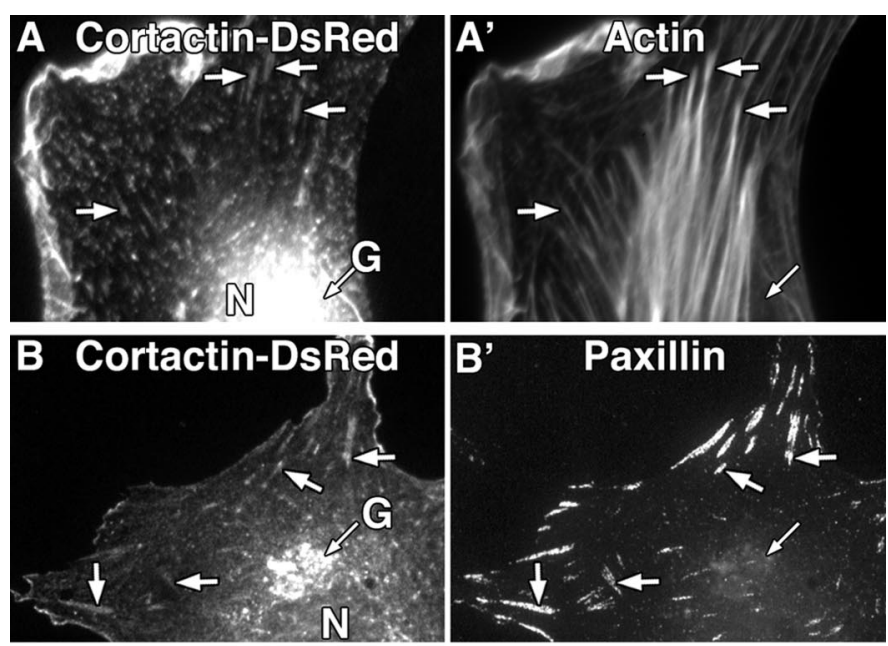

C

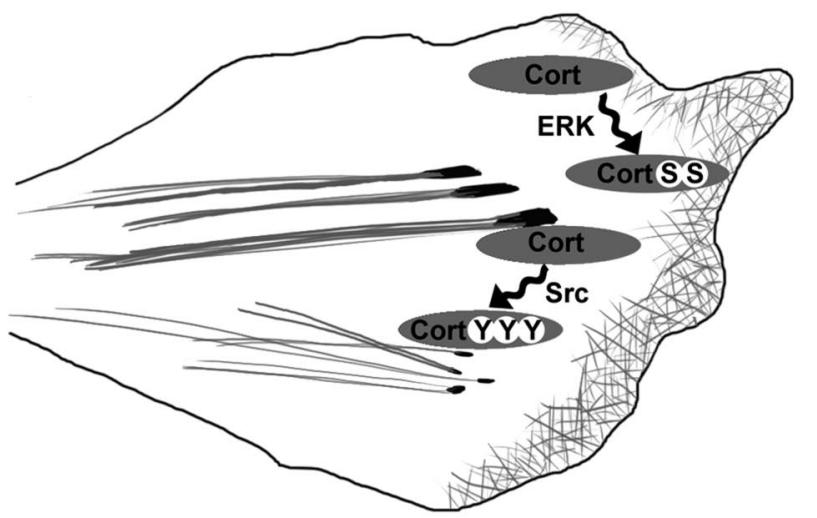

Fig. 6. Cortactin localizes to focal adhesions situated at the end of large actin stress fibers. $A-B^{\prime}$ : fluorescence images of fibroblasts expressing RFP-tagged CortWT $\left(A\right.$ and $B$ ) and stained for actin (rhodamine-phalloidin, $A^{\prime}$ ) or paxillin $\left(B^{\prime}\right)$. In addition to a prominent localization to lamellipodia and the Golgi, numerous cortactin-positive adhesion-like plaques ( $A$, arrows) can be seen oriented lengthwise throughout the cytoplasm, which coalign with the tips of large actin fibers $\left(A^{\prime}\right.$, arrows). The significant overlap with paxillin staining $\left(B^{\prime}\right.$, arrows) indicates that this cortactin localization represents focal adhesions. $G$, Golgi; $N$, nucleus. $C$ : cartoon depicting how distinct types of cortactin phosphorylation, ERK-mediated serine phosphorylation, and Src-mediated tyrosine phosphorylation could influence cell migration. Phosphorylation of cortactin on serine residues by ERK may promote the formation of dendritic branched actin networks that support lamellipodial extension. In contrast, Src-mediated tyrosine phosphorylation of cortactin appears to predominantly affect focal adhesion dynamics, resulting in increased focal adhesion turnover and a subsequent decrease in adhesion and stress fiber size. in vitro assays $(18,28)$, and these modifications are predicted to play an important role in the regulation of actin dynamics in living cells. Here, using expression of phospho-mutant forms of cortactin in cells, we provide a detailed, quantitative examination of the physiological effects of phospho-cortactin on actin-mediated processes in different pancreatic tumor cell types and fibroblasts. Incorporation of a variety of assays in our current study has allowed us to make significant observations regarding the mechanisms by which distinct forms of cortactin phosphorylation may regulate cell migration. Namely, although both tyrosine and serine phosphorylation of cortactin support lamellipodial extension and cell migration (Figs. 1 and 2 and supplemental Fig. S1), serine-phosphorylated cortactin may do so by promoting the assembly of dendritic actin networks (Fig. 3), while tyrosine phosphorylation of cortactin appears to regulate focal adhesion dynamics (Figs. 4 and 5; see cartoon, Fig. 6C).

Serine phosphorylation of cortactin promotes actin assembly in cells. Serine phosphorylation of cortactin has been reported to stimulate N-WASP-Arp2/3-mediated actin polymerization in vitro, and this stimulatory effect was prevented by subsequent tyrosine phosphorylation (18). More recently, another in vitro study using purified components demonstrated that tyrosine phosphorylation of cortactin does amplify the generation of actin barbed ends; however, this effect requires the adaptor protein Nck1 as an additional component of the actin assembly mixture. In addition, it is further enhanced by including WASP-interacting protein (WIP) (28). These latter components were not included in the earlier study. Our primary goal was to provide a useful extension of these in vitro observations to the context of living cells, something that has been lacking to date. To this end, we analyzed the effects of different cortactin phospho-mutants on the number, length, and girth of PIP5K1 $\alpha$-induced actin comet tails as an in vivo indicator of the assembly of actin networks. Indeed, our observations of a marked increase in comet numbers and dimensions in cells expressing CortSD are in strong support of the observations made in vitro. However, an increase in comet length was also observed in cells expressing CortWT and CortYE, indicating a complex interplay of cortactin phosphorylation in the regulation of actin dynamics within cells. Interestingly, unusually wide, bifurcated comets were observed at the light microscopy level in CortSD-expressing cells (Fig. $3 D^{\prime}$ ), and using electron microscopy, a wide base of actin nucleation that appeared to wrap around more than half of the vesicle membrane could be detected at the membrane interface. Such robust actin branching would be particularly useful at the leading edge of a migrating tumor cell to extend a lamellipodium forward.

Cortactin as a key regulator of focal adhesion dynamics? Cortactin has been implicated in the regulation of focal adhesion dynamics in migrating cells (1). Because expression of CortYE did not appear to alter actin assembly appreciably in vivo (Fig. 3) and Src-mediated phosphorylation is known to play a key role in regulating focal adhesion dynamics $(8,34)$, we tested whether tyrosine phosphorylation of cortactin may preferentially affect focal adhesion size and/or dynamics (Figs. 4 and 5). Here, we observed a marked increase in the size of focal adhesions in cells expressing either CortWT or CortYF, consistent with observations of others that cortactin promotes focal adhesion stability (1). Moreover, the marked increase in 
the size of focal adhesions in cells expressing CortYF (Fig. 4) suggests a role for cortactin tyrosine phosphorylation in focal adhesion disassembly. Indeed, observation of GFP-paxillin in living cells (Fig. 4) using TIRF microscopy revealed a substantial difference in focal adhesion turnover in cells expressing CortYE (turnover in 5-15 min) versus CortWT (turnover in 15-25 min) or CortYF (stable $>25 \mathrm{~min}$ ). Results from an assay using a distinct approach to measure focal adhesion dynamicsthe ability of focal adhesions to reassemble following microtubule-mediated disassembly-provided further support to these findings (Fig. 5).

All of these observations are consistent with an important role for cortactin phosphorylation in regulating focal adhesion dynamics, and in turn, lamellipodial protrusion. Furthermore, our findings are consistent with the premise that active Src (favoring tyrosine-phosphorylated cortactin and corresponding to CortYE) leads to smaller focal adhesions (8). Conversely, cells lacking Src family kinases or expressing a kinase-dead Src (corresponding to CortYF) have enlarged peripheral focal adhesions with impaired disassembly $(8,13,33)$. Thus, Srcmediated phosphorylation of cortactin may be important in regulating focal adhesion size and dynamics, and thereby modulate cell migration.

As illustrated in Fig. $6 C$, we propose that differential cortactin phosphorylation plays an important role in the regulation of two distinct processes, actin and focal adhesion dynamics, both of which can contribute to cell migration. How these processes are affected by differences in the phosphorylation status of cortactin is unclear, although alterations in proteinprotein interactions are likely to be involved. For example, serine phosphorylation of cortactin by ERK appears to allow for an increased association with N-WASP and facilitate branched actin assembly (18), whereas tyrosine phosphorylation of cortactin increases its association not only with Nck1 (28), but also with dynamin (37), an interaction which might contribute to focal adhesion disassembly (7). Based on the findings described here, in combination with those from other groups $(1,18,28)$, we predict that phospho-regulation of cortactin contributes to cell motility in two major ways. First, serine phosphorylation by ERK promotes the formation of branched actin networks, which supports lamellipodial formation and extension. Second, tyrosine phosphorylation by Src may also promote some actin assembly in vivo, although most conspicuously triggers turnover of focal adhesions and a reduction in the capping or anchoring of actin stress fibers at these structures.

In the future it will be important to determine how the specific phospho-forms of cortactin are regulated by different cellular stimulations, the interplay among the various types of cortactin phosphorylation, and the distinct effects of the various cortactin phospho-forms on the variety of cellular processes involving cortactin.

\section{ACKNOWLEDGMENTS}

The authors thank Dr. Heather M. Thompson for help in preparing the manuscript.

\section{GRANTS}

This work was supported by National Institutes of Health/National Cancer Institute Grant CA-104125 (to M. A. McNiven).

\section{REFERENCES}

1. Bryce NS, Clark ES, Leysath JL, Currie JD, Webb DJ, Weaver AM. Cortactin promotes cell motility by enhancing lamellipodial persistence. Curr Biol 15: 1276-1285, 2005.

2. Cameron LA, Svitkina TM, Vignjevic D, Theriot JA, Borisy GG. Dendritic organization of actin comet tails. Curr Biol 11: 130-135, 2001.

3. Campbell DH, Sutherland RL, Daly RJ. Signaling pathways and structural domains required for phosphorylation of EMS1/cortactin. Cancer Res 59: 5376-5385, 1999.

4. Cao H, Orth JD, Chen J, Weller SG, Heuser JE, McNiven MA. Cortactin is a component of clathrin-coated pits and participates in receptor-mediated endocytosis. Mol Cell Biol 23: 2162-2170, 2003.

5. Cao H, Weller S, Orth JD, Chen J, Huang B, Chen JL, Stamnes M, McNiven MA. Actin and Arf1-dependent recruitment of a cortactindynamin complex to the Golgi regulates post-Golgi transport. Nat Cell Biol 7: 483-492, 2005.

6. Daly RJ. Cortactin signalling and dynamic actin networks. Biochem $J$ 382: 13-25, 2004.

7. Ezratty EJ, Partridge MA, Gundersen GG. Microtubule-induced focal adhesion disassembly is mediated by dynamin and focal adhesion kinase. Nat Cell Biol 7: 581-590, 2005.

8. Frame MC, Fincham VJ, Carragher NO, Wyke JA. v-Src's hold over actin and cell adhesions. Nat Rev Mol Cell Biol 3: 233-245, 2002.

9. Frischknecht F, Way M. Surfing pathogens and the lessons learned for actin polymerization. Trends Cell Biol 11: 30-38, 2001.

10. Henley JR, McNiven MA. Association of a dynamin-like protein with the Golgi apparatus in mammalian cells. J Cell Biol 133: 761-775, 1996.

11. Huang C, Liu J, Haudenschild CC, Zhan X. The role of tyrosine phosphorylation of cortactin in the locomotion of endothelial cells. $J$ Biol Chem 273: 25770-25776, 1998.

12. Kim YW, Kern HF, Mullins TD, Koriwchak MJ, Metzgar RS. Characterization of clones of a human pancreatic adenocarcinoma cell line representing different stages of differentiation. Pancreas 4: 353-362, 1989.

13. Klinghoffer RA, Sachsenmaier C, Cooper JA, Soriano P. Src family kinases are required for integrin but not PDGFR signal transduction. EMBO J 18: 2459-2471, 1999.

14. Li Y, Tondravi M, Liu J, Smith E, Haudenschild CC, Kaczmarek M, Zhan X. Cortactin potentiates bone metastasis of breast cancer cells. Cancer Res 61: 6906-6911, 2001.

15. Lieber M, Mazzetta J, Nelson-Rees W, Kaplan M, Todaro G. Establishment of a continuous tumor-cell line (panc-1) from a human carcinoma of the exocrine pancreas. Int J Cancer 15: 741-747, 1975.

16. Lua BL, Low BC. Cortactin phosphorylation as a switch for actin cytoskeletal network and cell dynamics control. FEBS Lett 579: 577-585, 2005.

17. Martin KH, Jeffery ED, Grigera PR, Shabanowitz J, Hunt DF, Parsons JT. Cortactin phosphorylation sites mapped by mass spectrometry. J Cell Sci 119: 2851-2853, 2006.

18. Martinez-Quiles N, Ho HY, Kirschner MW, Ramesh N, Geha RS. Erk/Src phosphorylation of cortactin acts as a switch on-switch off mechanism that controls its ability to activate N-WASP. Mol Cell Biol 24: 5269-5280, 2004.

19. Mizutani K, Miki H, He H, Maruta H, Takenawa T. Essential role of neural Wiskott-Aldrich syndrome protein in podosome formation and degradation of extracellular matrix in $s r c$-transformed fibroblasts. Cancer Res 62: 669-674, 2002.

20. Orth JD, Krueger EW, Cao H, McNiven MA. The large GTPase dynamin regulates actin comet formation and movement in living cells. Proc Natl Acad Sci USA 99: 167-172, 2002.

21. Orth JD, Krueger EW, Weller SG, McNiven MA. A novel endocytic mechanism of epidermal growth factor receptor sequestration and internalization. Cancer Res 66: 3603-3610, 2006.

22. Orth JD, McNiven MA. Dynamin at the actin-membrane interface. Curr Opin Cell Biol 15: 31-39, 2003.

23. Patel AS, Schechter GL, Wasilenko WJ, Somers KD. Overexpression of EMS1/cortactin in NIH3T3 fibroblasts causes increased cell motility and invasion in vitro. Oncogene 16: 3227-3232, 1998.

24. Rajasekaran SA, Gopal J, Espineda C, Ryazantsev S, Schneeberger EE, Rajasekaran AK. HPAF-II, a cell culture model to study pancreatic epithelial cell structure and function. Pancreas 29: e77-e83, 2004.

25. Rozelle AL, Machesky LM, Yamamoto M, Driessens MH, Insall RH, Roth MG, Luby-Phelps K, Marriott G, Hall A, Yin HL. Phosphatidyl- 
inositol 4,5-bisphosphate induces actin-based movement of raft-enriched vesicles through WASP-Arp2/3. Curr Biol 10: 311-320, 2000.

26. Sipos B, Moser S, Kalthoff H, Torok V, Lohr M, Kloppel G. A comprehensive characterization of pancreatic ductal carcinoma cell lines: towards the establishment of an in vitro research platform. Virchows Arch 442: 444-452, 2003.

27. Tan MH, Nowak NJ, Loor R, Ochi H, Sandberg AA, Lopez C, Pickren JW, Berjian R, Douglass HO Jr, Chu TM. Characterization of a new primary human pancreatic tumor line. Cancer Invest 4: 15-23, 1986.

28. Tehrani S, Tomasevic N, Weed S, Sakowicz R, Cooper JA. Src phosphorylation of cortactin enhances actin assembly. Proc Natl Acad Sci USA 104: 11933-11938, 2007.

29. Timpson P, Wilson AS, Lehrbach GM, Sutherland RL, Musgrove EA, Daly RJ. Aberrant expression of cortactin in head and neck squamous cell carcinoma cells is associated with enhanced cell proliferation and resistance to the epidermal growth factor receptor inhibitor gefitinib. Cancer Res 67: 9304-9314, 2007.

30. Van Damme H, Brok H, Schuuring-Scholtes E, Schuuring E. The redistribution of cortactin into cell-matrix contact sites in human carcinoma cells with 11q13 amplification is associated with both overexpression and posttranslational modification. J Biol Chem 272: 7374-7380, 1997.

31. Weaver AM, Young ME, Lee WL, Cooper JA. Integration of signals to the Arp2/3 complex. Curr Opin Cell Biol 15: 23-30, 2003.

32. Webb DJ, Brown CM, Horwitz AF. Illuminating adhesion complexes in migrating cells: moving toward a bright future. Curr Opin Cell Biol 15: 614-620, 2003.

33. Webb DJ, Donais K, Whitmore LA, Thomas SM, Turner CE, Parsons JT, Horwitz AF. FAK-Src signalling through paxillin, ERK and MLCK regulates adhesion disassembly. Nat Cell Biol 6: 154-161, 2004.

34. Webb DJ, Parsons JT, Horwitz AF. Adhesion assembly, disassembly and turnover in migrating cells - over and over and over again. Nat Cell Biol 4: E97-100, 2002.

35. Weed SA, Parsons JT. Cortactin: coupling membrane dynamics to cortical actin assembly. Oncogene 20: 6418-6434, 2001.

36. Wu H, Reynolds AB, Kanner SB, Vines RR, Parsons JT. Identification and characterization of a novel cytoskeleton-associated pp $60^{s r c}$ substrate. Mol Cell Biol 11: 5113-5124, 1991.

37. Zhu J, Yu D, Zeng XC, Zhou K, Zhan X. Receptor-mediated endocytosis involves tyrosine phosphorylation of cortactin. J Biol Chem 282: $16086-$ 16094, 2007.

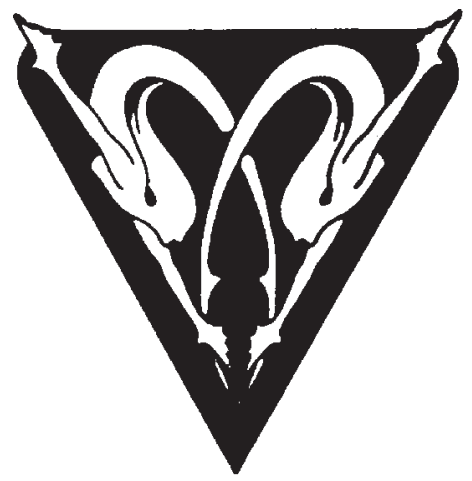

